\title{
Une diode électroluminescente à une molécule
}

\author{
Guillaume SCHULL, Fabrice SCHEURER et Gaël REECHT \\ Institut de Physique et Chimie des Matériaux de Strasbourg, \\ UMR 7504 (CNRS-Université de Strasbourg), 67034 Strasbourg, France. \\ schull@unistra.fr
}

Un premier composant de taille moléculaire combinant des propriétés électroniques et photoniques a été réalisé. Pour ce faire, un brin unique d'un polymère conducteur (polythiophène) a été étiré entre une surface métallique et la pointe effilée d'un microscope à effet tunnel (STM) fonctionnant à basse température (4K). Lorsqu'une tension de l'ordre de $2 \mathrm{~V}$ est appliquée entre ces deux électrodes, le courant électrique traversant la molécule génère une émission de lumière. Cette émission est liée à la recombinaison entre un électron injecté dans la molécule depuis la pointe avec un trou injecté depuis l'échantillon. Le mécanisme de luminescence ainsi que la dépendance en tension de l'émission montre que la jonction se comporte comme une diode électroluminescente (LED) à une molécule.

Les composants de l'électronique organique

\section{L'intérêt des composants moléculaires}

L'électronique organique vise à remplacer les composants électroniques basés sur les semi-conducteurs traditionnels, par des composés organiques. Des gains substantiels sont attendus, notamment en termes d'énergie lors de la production ou du fonctionnement des composants, ainsi qu'en termes de flexibilité mécanique. En parallèle, de nouvelles approches technologiques sont nécessaires afin de permettre une miniaturisation plus importante des composants. Dans ce cadre, la réalisation de circuits où chaque composant serait une seule molécule est perçue comme l'étape ultime alliant électronique organique et petites échelles. Les progrès techniques des dernières décennies ont permis la réalisation de jonctions électriques dont la partie la plus résistive est composée d'une molécule unique. Celle-ci est généralement connectée par deux de ses extrémités à des électrodes métalliques où est injecté et/ou collecté le courant électrique. Si, de cette manière, certains composants moléculaires ont été réalisés (fils, interrupteurs...) la perspective de composants à cette échelle combinant des propriétés électroniques et optiques n'a que récemment été évoquée sous le terme d'optoélectronique moléculaire. Dans ce cadre la réalisation d'une diode électroluminescente moléculaire (Reecht et coll. [1]) constitue un progrès important.

\section{Des premiers résultats prometteurs}

Pour réaliser ce type de composants il faut être capable d'adresser simultanément les propriétés électroniques et optiques d'une seule molécule. Un résultat pionnier dans ce cadre a été rapporté en 2003 par Qiu et coll. [2] qui ont pu exciter électriquement la fluorescence d'une molécule unique avec des électrons tunnel injectés depuis la pointe d'un STM (figure 1a). Dans cette expérience la molécule est déposée sur une surface métallique recouverte d'une fine couche d'isolant. De cette manière les états électroniques de la molécule sont découplés de ceux de la pointe (par le vide) et de l'échantillon (par l'isolant). On peut ainsi considérer que la molécule est « partiellement isolée » des électrodes ce qui est une condition nécessaire à la fluorescence. Toutefois, afin de se rapprocher d'une future configuration d'utilisation, un contact direct entre électrodes et molécule est souhaitable comme représenté en figure 1b. On forme ainsi un véritable circuit électrique. De récentes expériences ont été menées dans ce sens, et ont révélé les propriétés d'électroluminescence d'une molécule unique de C60 en contact direct avec deux électrodes de cuivre [3]. Cette étude montre que le bruit de grenaille du courant traversant la molécule est ici responsable de l'émission de lumière. Si les spectres optiques obtenus révèlent également l'influence de plasmons de surface localisés entre la surface métallique et la pointe STM, ils ne montrent aucun signe d'une transition électronique intrinsèque à la molécule. Ce phénomène dit d'extinction de la fluorescence (quenching) est bien connu, et s'explique par le rapide transfert de charges entre molécule et électrodes en contact direct.

\section{Comment dès lors combiner contact direct et fluorescence au sein d'une même jonction moléculaire?}

Pour ce faire nous nous sommes inspirés de travaux publiés par Lafferentz et coll. [4] qui ont montré qu'il est possible de relever un long fil moléculaire d'une surface 

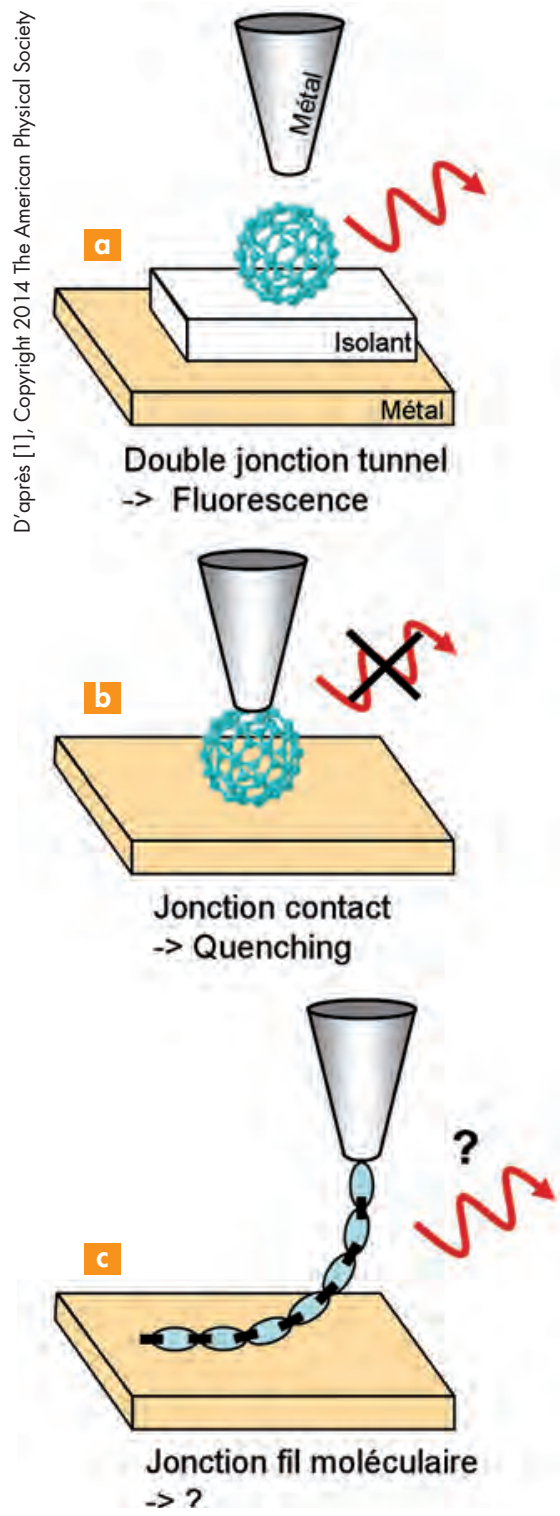

Figure 1. Electroluminescence d'une molécule unique par STM pour le cas (a) d'une double jonction tunnel, (b) d'une jonction contact et (c) d'un fil moléculaire suspendu entre pointe et échantillon.

métallique en attachant l'une de ses extrémités à la pointe d'un STM. Le fil moléculaire est ainsi en contact direct avec la pointe et l'échantillon, tout en présentant une partie (de quelques nanomètres de long) qui est librement suspendue entre les deux (figure 1c). Le but de notre travail a consisté à sonder les propriétés d'électroluminescence de ce type de structure, avec l'espoir que la partie centrale du fil moléculaire se comporte comme une molécule isolée permettant de retrouver des spectres de luminescence intrinsèque au fil. Ci-dessous nous décrivons les méthodes permettant de réaliser et de manipuler ces fils moléculaires ainsi que celles permettant de sonder leurs propriétés optoélectroniques, nous rapportons les principaux résultats expérimentaux obtenus et discutons les mécanismes mis en évidence.

\section{Réalisation d'une diode électroluminescente à une molécule}

\section{Réalisation des fils moléculaires}

Afin de garantir une très bonne stabilité des jonctions moléculaires et de faciliter la manipulation des fils de polythiophène, les expériences ont été réalisées dans des conditions d'ultra-haut vide ( $10^{-11} \mathrm{mbar}$ ) et à des températures cryogéniques (de l'ordre de $5 \mathrm{~K}$ ). Cela implique également un certain nombre de contraintes, notamment pour ce qui est du dépôt des molécules sur des surfaces atomiquement plates d'or. Dans ces conditions, les dépôts sont en général obtenus par sublimation sous vide des molécules depuis la phase solide. Pour des fils de plusieurs nanomètres de long comme ceux visés par l'expérience, cela implique des températures extrêmement élevées qui conduiraient à la dégradation des fils moléculaires. Nous avons ainsi opté pour une méthode alternative consistant à utiliser des briques élémentaires des fils moléculaires visés nécessitant des températures moins élevées pour leur sublimation, et a générer dans une deuxième étape une réaction de polymérisation sur la surface. Cette méthode a ainsi permis l'obtention de longs fils moléculaires (plusieurs dizaines de nanomètres dans certains cas) dont on peut visualiser un exemple sur l'image STM de la figure $2 a$.

\section{Détacher un fil moléculaire et mesurer sa conductance}

On peut attacher une des extrémités d'un tel fil à la pointe métallique du STM. Pour ce faire, celle-ci est positionnée en regard de l'extrémité du fil choisi et approchée de celui-ci jusqu'à formation d'un contact, puis relevée progressivement, de façon à laisser quelques

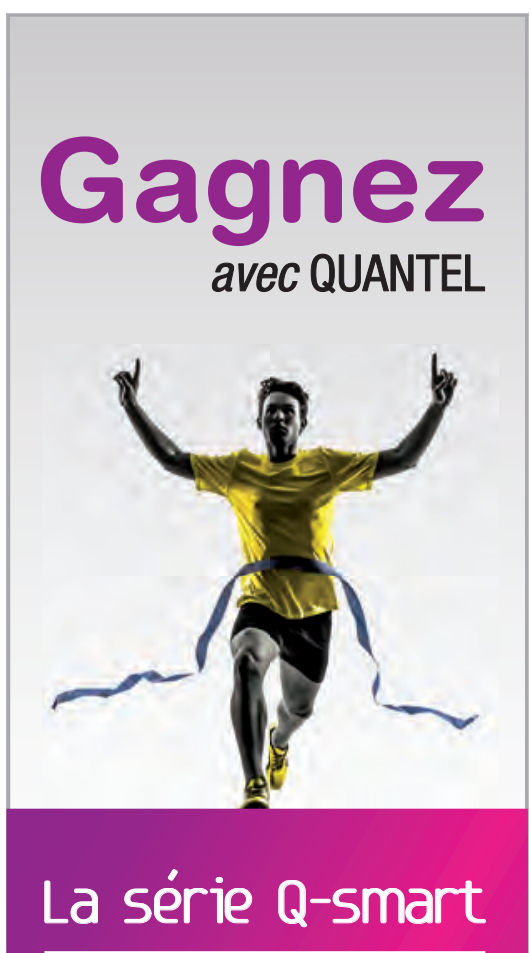

Les nouveaux lasers Nd:YAG pulsés par Quantel

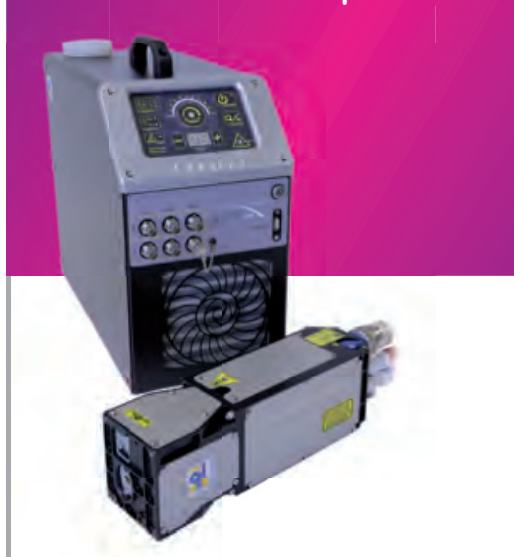

\section{La qualité conçue pour durer}

\section{(q) Quantel}

quantel@quantel-laser.com www.quantel-laser.com 

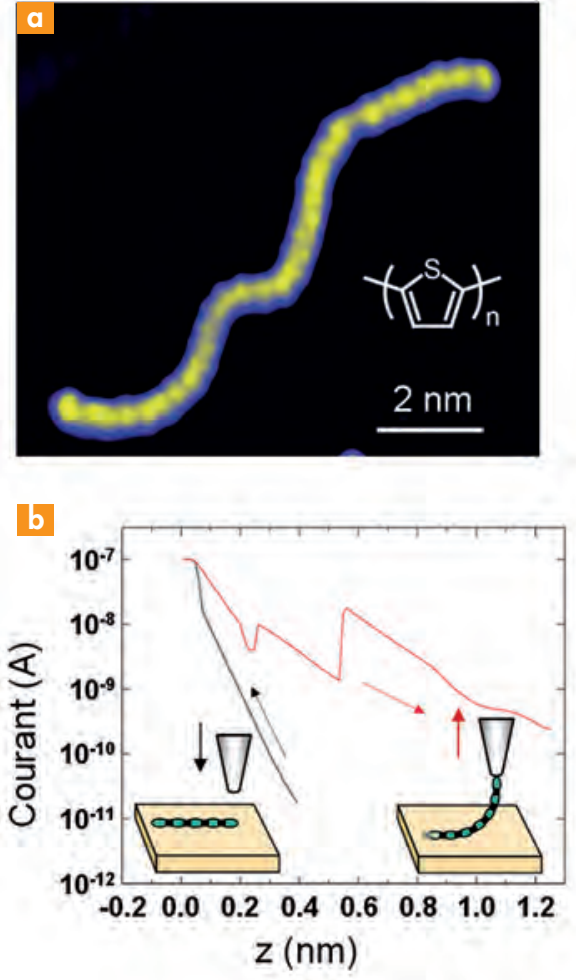

nanomètres ( 3 à 5) de fil suspendu entre pointe et échantillon. L'enregistrement simultané du courant traversant la jonction lors de cette procédure est présenté à la figure $2 b$. Lors de l'approche de la pointe (courbe noire) le courant augmente exponentiellement, ce qui est attendu pour une jonction tunnel (absence de contact entre pointe et molécule), jusqu'à la formation du contact marquée par un saut brutal de courant à faible distance $(z$ $0 \mathrm{~nm}$ ). Lors de la rétraction de la pointe (courbe rouge) le courant est plus intense de plusieurs ordres de grandeur, du fait de la bonne conductance du polythiophène. Par ailleurs, on notera plusieurs sauts de courant importants, associés aux détachements successifs des briques élémentaires du fil moléculaire de la surface. Ces différents aspects attestent de la réussite de la procédure permettant de suspendre les fils dans la jonction, et nous informent sur les propriétés de transport de charges au sein d'une jonction contenant un fil unique de polythiophène. Une fois cette configuration obtenue, il convient de détecter les photons susceptibles d'être émis (figure 2c) par le passage du courant.
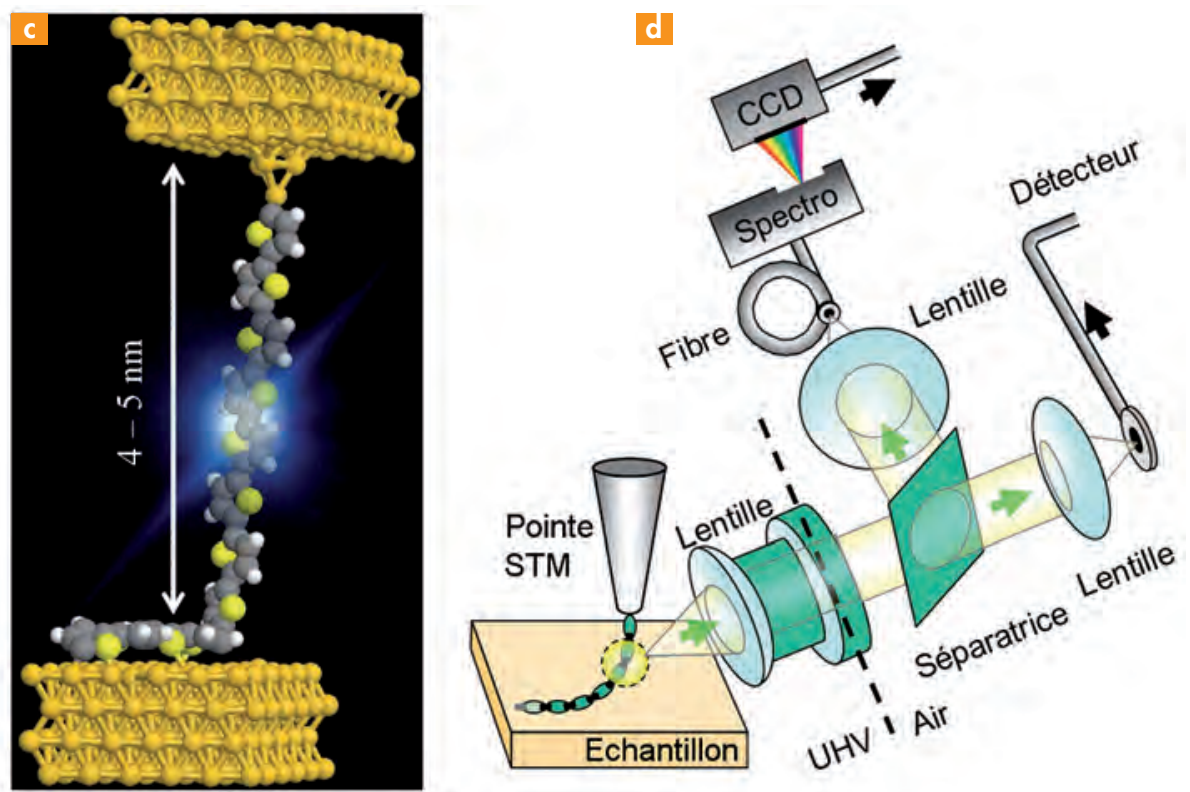

Figure 2. (a) Image STM d'un fil moléculaire de polythiophène sur une surface d'Au(1 1 1). (b) Évolution du courant traversant la jonction lors de la procédure consistant à suspendre le fil moléculaire entre pointe ef échantillon. (c) Vue artistique de l'électroluminescence de la jonction contenant le fil moléculaire. (d) Schéma du dispositif expérimental permettant la collection de la lumière émise à la jonction d'un microscope à effet tunnel.

\section{Détecter l'électroluminescence d'un fil moléculaire}

Pour analyser les photons émis, il faut ménager un accès optique jusqu'à la jonction, ce qui est rendu difficile par l'édifice expérimental permettant de travailler sous vide et basse température. Une lentille est fixée de telle sorte que la jonction se situe à son point focal pour collecter les photons émis (la profondeur de champ correspond à un volume d'environ $2 \mathrm{~mm}^{3}$ ). La lumière collimatée est dirigée en faisceau parallèle vers l'extérieur de l'enceinte au travers de fenêtres disposées sur les écrans thermiques refroidis à I'hélium et à l'azote liquide, puis détectée à l'aide d'un photo-détecteur ou d'une caméra CCD associée à un spectromètre (figure $2 d$ ).

\section{Electro-fluorescence d'un fil moléculaire}

Lorsqu'une tension ( $V \sim 2 \mathrm{eV}$ ) est appliquée entre la pointe et l'échantillon d'un STM une lumière est émise, même en l'absence de structures organiques dans la jonction. Ce phénomène est lié à des électrons dit inélastiques qui perdent leur énergie en traversant la jonction tunnel. L'énergie perdue permet l'émission d'un photon dont l'énergie est toujours inférieure à celle de l'électron qui l'a créé (hu<el). Notons également que pour des électrodes en or ou en argent, ce mécanisme est efficacement amplifié par des plasmons de surface localisés à la jonction. En figure $3 a$ (spectre $\Gamma$ ) nous représentons un spectre d'électroluminescence d'une jonction or-or pour une tension de $3 \mathrm{~V}$. On y observe un pic principal vers 1,55 eV ainsi que plusieurs épaulements à des énergies comprises entre $1,2 \mathrm{eV}$ (limite de détection de la CCD) et 2,3 eV (limite à haute énergie des plasmons de surface de l'or). Il a été démontré que la position et le nombre des pics et/ou épaulements dans ces spectres peuvent varier de manière significative pour un même matériau en fonction de la forme et du rayon de courbure de la pointe STM (à l'échelle de quelques dizaines de nanomètres). Pour cette même pointe STM nous représentons également en figure $3 a$ des spectres obtenus avec une molécule de polythiophène relevée de 4 à $5 \mathrm{~nm}$ (figure 2c). Ces spectres semblent indiquer un déplacement vers les hautes énergies $(\sim 1,7 \mathrm{eV})$ du pic principal. Toutefois, les pics et épaulements identifiés dans le spectre de la jonction oror sont également présents aux mêmes énergies dans les spectres de la molécule. 
Cette observation (maintes fois répétée) montre que les plasmons localisés à la jonction pointe-surface amplifient également le processus d'émission de photons dans le cas de la jonction moléculaire. Afin de déterminer ce qui est directement imputable à la présence du fil moléculaire au sein de la jonction, il convient de corriger les spectres de l'influence des plasmons. Pour cela il suffit de normaliser les spectres d'émission de la jonction moléculaire par
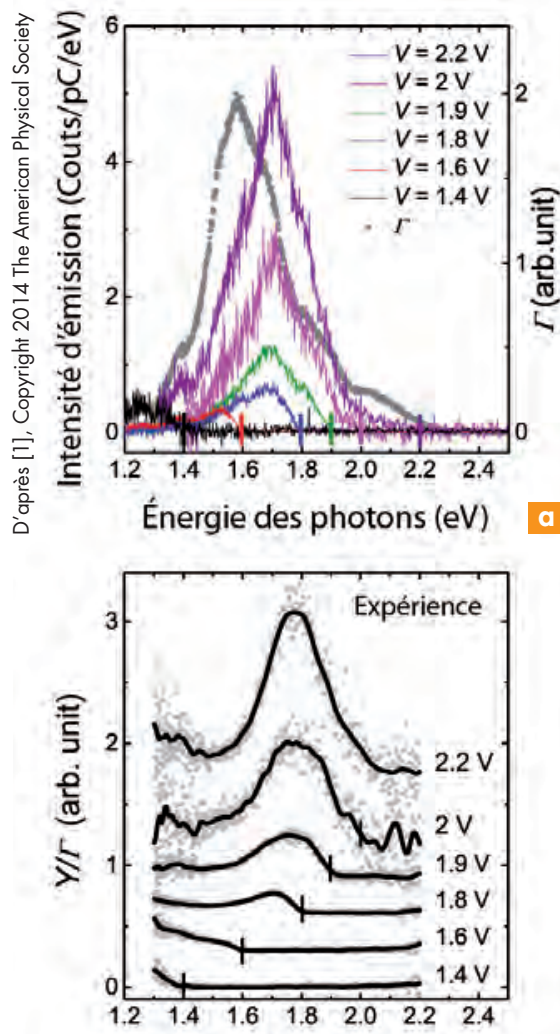

Énergie des photons (eV)

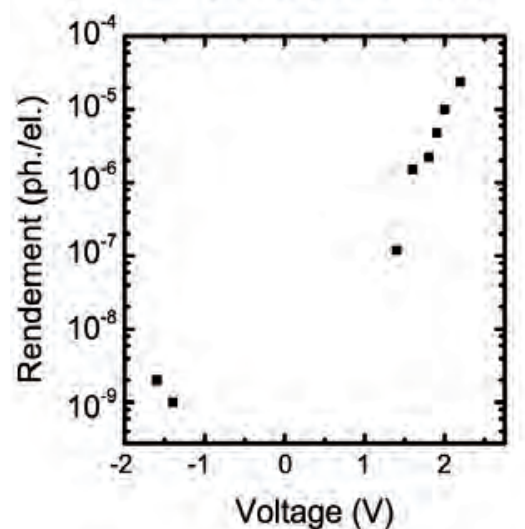

Figure 3. Spectres optiques bruts (a) et corrigés de I'influence des plasmons (b) de la jonction contenant le fil moléculaire en fonction de la tension pointeéchantillon appliquée. (c) Rendement de l'émission (en photons/électrons) en fonction de la tension. celui obtenu, avec la même pointe, pour la jonction or-or. Les spectres corrigés de I'influence des plasmons sont représentés en figure $3 b$ en fonction de la tension. Comme prévu, l'énergie des photons émis ne dépasse pas celle des électrons traversant la jonction ( $\mathrm{hu}<\mathrm{eV}$ ). Pour les tensions croissantes on observe également l'apparition progressive d'un pic centré à environ 1,8 eV dont l'énergie ne varie pas pour les tensions plus élevées. Cette observation, comme nous allons le démontrer dans la suite, suggère une transition entre deux états électroniques au sein de la jonction, qui sont directement liés à la présence du fil moléculaire entre pointe et échantillon Enfin, l'intensité d'émission de la jonction moléculaire dépend fortement du signe de la tension appliquée ou, en d'autres termes, du sens dans lequel le courant traverse la jonction (figure 3c). L'amplification par les plasmons étant indépendante du signe de la tension, cette observation souligne une fois encore le rôle déterminant du fil moléculaire dans l'émission lumineuse.

\section{Mécanisme d'émission}

Les résultats expérimentaux décrits ci-dessus suggèrent que, lorsque le fil de polythiophène est introduit entre pointe et surface, ses orbitales moléculaires influencent fortement le passage du courant, via les canaux de décroissance inélastique et donc, in fine, le spectre d'émission de la jonction. Pour confirmer cette interprétation et comprendre comment ce phénomène fonctionne nous avons établi le modèle simple représenté à la figure 4. Le schéma en (a) décrit l'état de la jonction à tension nulle, notamment la position en énergie de l'orbitale inoccupée la plus basse $(\mathrm{LUMO}=$ lowest unoccupied molecular orbital) et de l'orbitale occupée la plus haute (HOMO = highest occupied molecular orbital) du fil moléculaire par rapport aux niveaux de Fermi respectifs des électrodes. La position en énergie des orbitales est un paramètre d'ajustement du modèle, par ailleurs en excellent accord avec des résultats issus de calculs numériques. Le schéma en (b) montre comment la configuration énergétique de la jonction évolue lorsqu'une tension d'échantillon de $2 \mathrm{~V}$ est appliquée

\section{Imagine ( Xaptic}

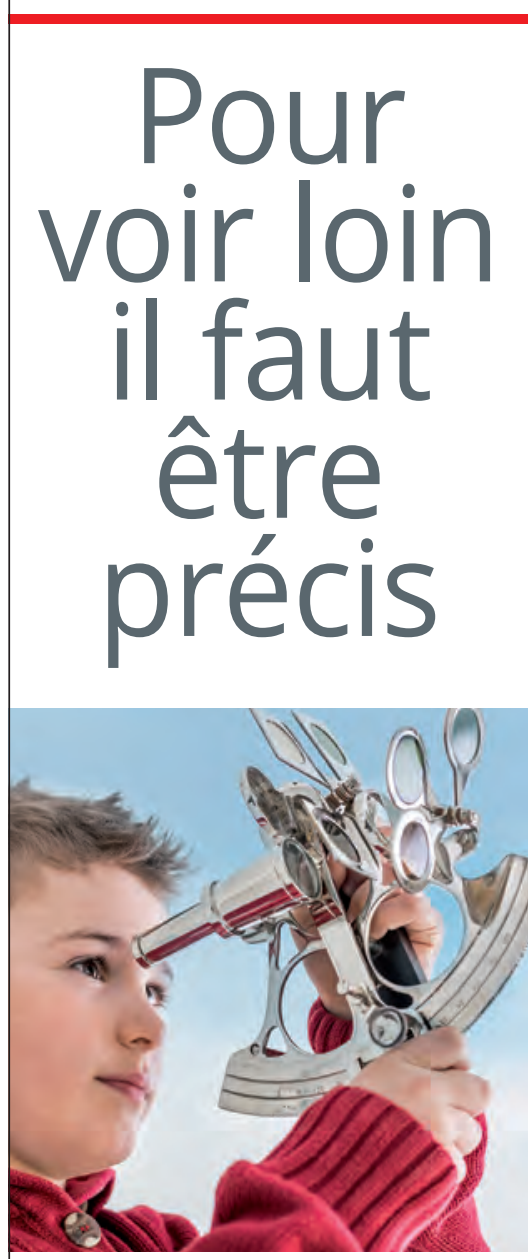

\section{ANALYSEUR DE FRONT D'ONDE \\ MIROIR DÉFORMABLE OPTIQUE ADAPTATIVE}

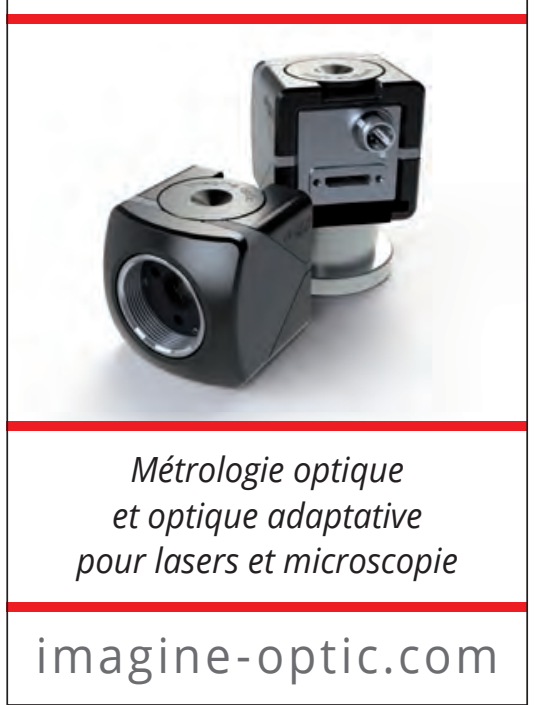



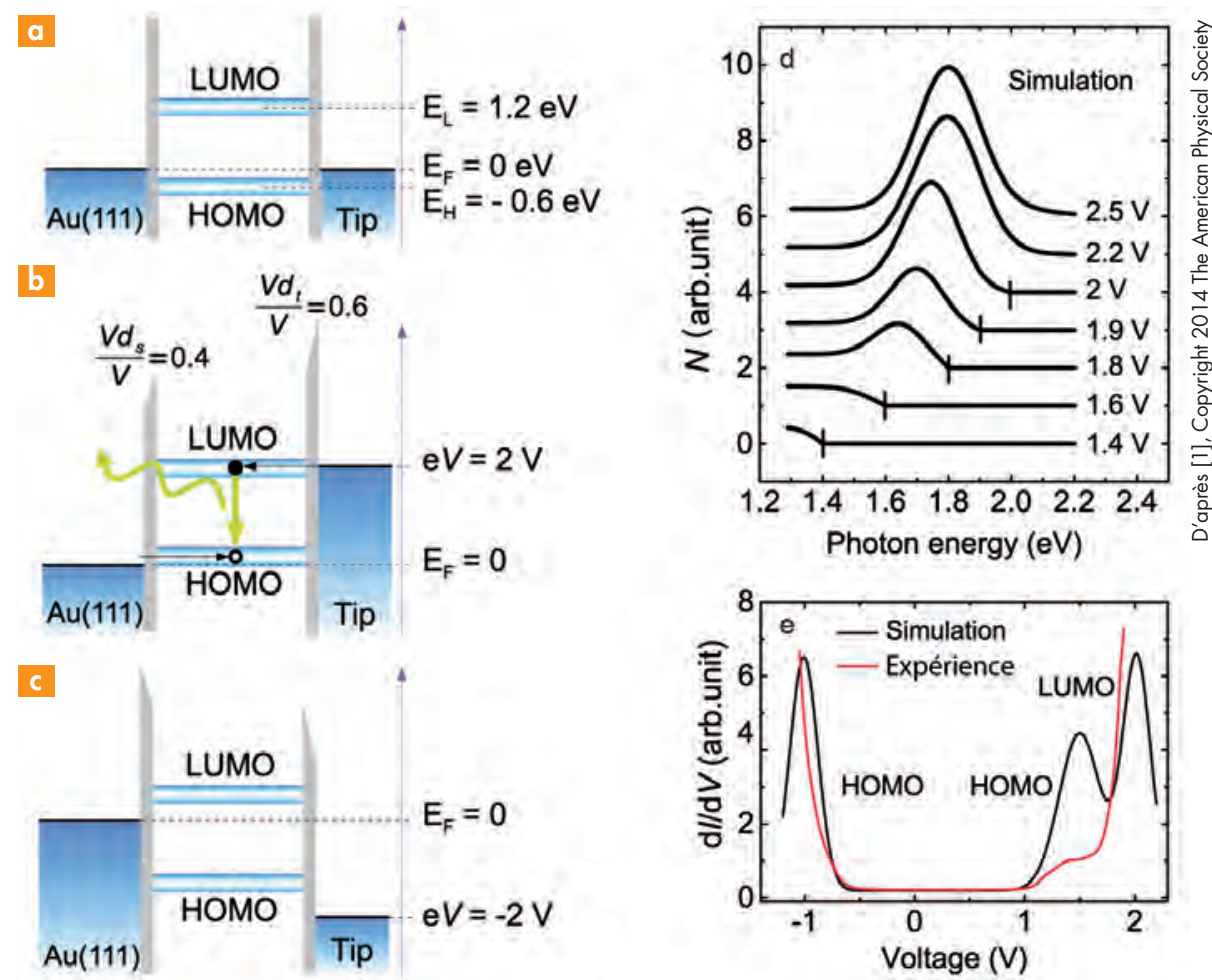

Figure 4. (a-c) Modèles illustrant le mécanisme d'émission. Configuration énergétique de la jonction à tension nulle (a), à une tension d'échantillon de $2 \mathrm{~V}(b)$ et de $-2 \mathrm{~V}(\mathrm{c})$. (d) Spectres optiques simulés pour des tensions d'échantillon allant de 1,4 V à 2,5 V déduits du modèle en (b). (e) Caractéristiques courant-tension $(I(V))$ expérimentales (trait rouge) et simulées (noir) d'une jonction contenant un fil moléculaire suspendu entre pointe et échantillon pour les mêmes paramètres ajustables que ceux utilisés pour simuler les spectres optiques en (d).

entre pointe et échantillon. Le niveau de Fermi de la pointe est alors résonnant avec la LUMO dans laquelle il devient possible d'injecter des électrons. Dans le même temps, le niveau de Fermi de l'électrode devient résonnant avec la HOMO de la molécule ce qui permet d'en extraire les électrons ( $d$ 'y injecter des trous). Cette configuration énergétique n'est rendue possible que par la géométrie très particulière des contacts entre le fil et ses électrodes conduisant à une chute de la tension répartie aux deux interfaces. Il est dès lors possible aux électrons injectés dans la LUMO de décroître vers la HOMO de la molécule en produisant un photon. La figure $4 d$ présente l'évolution en fonction de la tension des spectres d'émission simulés sur la base de ce mécanisme. Comme dans l'expérience (figure $3 b$ ) on observe l'apparition progressive d'un pic centré à environ 1,8 eV dont l'énergie ne varie pas avec la tension. Sur la base de ce modèle il est également possible de montrer pourquoi la jonction n'émet pas pour les tensions négatives : en figure $4 c$ nous représentons la configuration énergétique de la jonction pour une tension d'échantillon de $-2 \mathrm{~V}$. La faible chute de potentiel à l'interface échantillon-molécule ne permet pas l'injection d'électrons dans la LUMO à cette tension, empêchant le processus de fluorescence. Cette différence de comportement en fonction du signe de la tension est due à l'asymétrie des interfaces molécule-électrodes, qui peut être visualisée en figure $4 c$, et qui se traduit par une plus grande résistance de l'interface pointe-molécule. Les paramètres ajustables sont donc les positions et largeurs en énergie des niveaux moléculaires, et le rapport de la chute de potentiel aux interfaces fil-pointe et fil-surface.II est possible de trouver avec le même jeu de paramètres une bonne description, à la fois de l'évolution des spectres de luminescence avec la tension appliquée, et des caractéristiques courant-tension (I/V) de la jonction (figure 4e), ce qui conforte le modèle proposé.

\section{Vers des sources extrêmement ponctuelles}

Ces résultats montrent qu'il est possible d'associer, au sein d'une même jonction moléculaire, fluorescence et couplage fort entre molécule et électrodes. Pour cela nous avons dû utiliser des structures organiques présentant de bonnes capacités de conduction et suffisamment allongées (cas des polymères conjugués) afin que la partie suspendue entre les électrodes se retrouve partiellement " isolée » des électrodes. La configuration particulière des interfaces molécule-électrodes (asymétrie des interfaces molécule-pointe et molécule-surface) est responsable de la forte variation de l'intensité d'émission (3 à 4 ordres de grandeur) entre tension positive et négative. La combinaison d'une luminescence liée à des recombinaisons électron-trou avec une forte asymétrie d'émission avec la polarité rappelle le comportement des diodes électroluminescentes macroscopiques. Le fait que des mécanismes équivalents puissent être obtenus à l'échelle d'une seule jonction moléculaire ouvre la voie à la réalisation de composants optoélectroniques aux échelles nanométriques. Ce type de structures pourrait notamment servir de source d'excitation électrique extrêmement ponctuelle et bien définie de plasmons-polaritons permettant le transfert d'informations au sein de guides d'onde plasmoniques.

\section{Références}

[1] G. Reecht, F. Scheurer, V. Speisser, Y.J. Dappe, F. Mathevet, G. Schull, Electroluminescence of a polythiophene molecular wire suspended between a metallic surface and the tip of a scanning tunneling microscope, Phys. Rev. Lett. 112 , 047403 (2014).

[2] X.H. Qiu, G.V. Nazin, W. Ho, Vibrationally resolved fluorescence excited with submolecular precision, Science 299, 542 (2003).

[3] N.L. Schneider, J.T. Lü, M. Brandbyge, R. Berndt, Light emission probing quantum shot noise and charge fluctuations at a biased molecular junction, Phys. Rev. Lett. 109, 186601 (2012).

[4] L. Lafferentz, F. Ample, H. Yu, S. Hecht, C. Joachim, L. Grill, Conductance of a single conjugated polymer as a continuous function of its length, Science 323, 1193 (2009). 